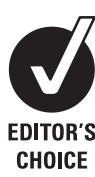

${ }^{1}$ Chronic Disease and Health Promotion Department, Prevention of Blindness and Deafness, World Health Organization, Geneva, Switzerland; ' ${ }^{2}$ School of Medicine, University of California, San Francisco, California, USA

Correspondence to: Dr S P Mariotti, World Health Organization, 20 avenue appia Geneva, Switzerland; mariottis@who.int

Accepted 3 December 2008 Published Online First 19 December 2008

\title{
Trachoma: global magnitude of a preventable cause of blindness
}

\author{
S P Mariotti, ${ }^{1}$ D Pascolini, ${ }^{1}$ J Rose-Nussbaumer ${ }^{2}$
}

\begin{abstract}
Objectives: Trachoma is the leading cause of infectious blindness worldwide. It is known to be highly correlated with poverty, limited access to healthcare services and water. In 2003, the WHO estimated that 84 million people were suffering from active trachoma, and 7.6 million were severely visually impaired or blind as a result of trachoma: this study provides an updated estimate of the global prevalence of trachoma based on the most recent information available.
\end{abstract}

Methods: A literature search of recent published and unpublished surveys in the 57 endemic countries was carried out: the result of surveys that used the WHO trachoma grading system and additional information from regional and country experts served as a basis to determine the prevalence of trachoma in each country.

Results: Population-based surveys provided recent information for 42 out of 57 endemic countries. 40.6 million people are estimated to be suffering from active trachoma, and 8.2 million are estimated to have trichiasis.

Conclusions: The current estimate of prevalence of trachoma is lower than the previous WHO estimates: this can be explained by the success in implementing control strategy, by more accurate data, as well as by socioeconomic development in endemic countries.

Trachoma is the leading cause of infectious blindness worldwide: ${ }^{1}$ it is caused by ocular infections with Chlamydia trachomatis that might result in chronic inflammation of the eyelids. These infections occur mainly in children, peaking around age 1-5 and declining thereafter. This chronic inflammation of the eyelids produces scarring of the conjunctiva that can subsequently cause entropion trichiasis, resulting in inturned eyelashes. The inturned eyelashes as well as other alterations of the eye, such as lacrimal function and corneal limbus, harm the cornea causing severe pain, corneal opacity and consequent vision loss. ${ }^{2}$

The disease is known to be highly correlated with poverty, lack of personal and community hygiene, limited access to healthcare and water. It is prevalent in Africa, Asia and some parts of Latin America, the Middle East and the Western Pacific. ${ }^{3}$ Active trachoma affects mainly women and children, and cicatricial trachoma affects on average women three times more than men likely secondary to their prolonged contact with infected children. ${ }^{45}$ In 2003, the WHO estimated that 84 million people were suffering from active trachoma, and 7.6 million were severely visually impaired or blind as a result of trachoma. ${ }^{6}$

When visual impairment occurs during the productive years of life, it results in economic hardship for families and communities: it has been estimated that in countries ranked with a low Human Development Index (http://hdr.undp.org/ en/statistics/) the disease could result in a total of 2.9 billion dollars in lost productivity each year. ${ }^{7}$

In 1997, the WHO established the Alliance for Global Elimination of Trachoma (GET) by the year 2020.8 The elimination strategy has been summarised with the acronym SAFE, which stands for Surgery for trichiasis, Antibiotics, Facial cleanliness and Environmental change, such as clean water and latrines. ${ }^{9} 10$

This paper provides an updated estimate of the global magnitude of trachoma based on the most recent information available. The knowledge of the prevalence of trachoma at country and global level is essential for the planning and for the implementation of the interventions needed to eliminate this preventable cause of blindness and ultimately for the achievement of the WHO World Health Assembly Resolution for the Global Elimination of Trachoma by 2020 WHA 51:11 (available at http://www.who.int/blindness/causes/WHA51.11/ en/index.html).

\section{METHODS}

\section{Epidemiological data}

An extensive literature search was carried out for published and unpublished literature from the year 2000 onwards regarding the prevalence of trachoma in the 57 countries known to be endemic. Published data were searched using Medline with the keywords "trachoma prevalence" up to 6 July 2007. The studies selected were population-based surveys that gave a description of sample design and plan, sample size, response rate and examination methods. Unpublished data were gathered in part during the Eleventh Meeting of the WHO Alliance for the Global Elimination of Trachoma by $2020^{11}$ from the reports and presentations of country experts. Additional data were obtained by contacting experts from countries and regions or researchers in academic institutions.

The majority of the surveys used an established WHO protocol for sampling populations to determine prevalence of trachoma (available at http://www.who.int/blindness/prevalence_protocol_ english.pdf). All surveys selected assessed trachoma using the simplified WHO trachoma grading system (http://www.WHO.int/Blindness/Causes/Priority/ en/index2.html): Trachomatous InflammationFollicular (TF) was defined as the presence of five or more follicles in the upper tarsal conjunctiva; Trachomatous Inflammation-Intense (TI) was defined as pronounced inflammatory thickening of the tarsal conjunctiva that obscures more than half of the normal deep tarsal vessels; Trachomatous 

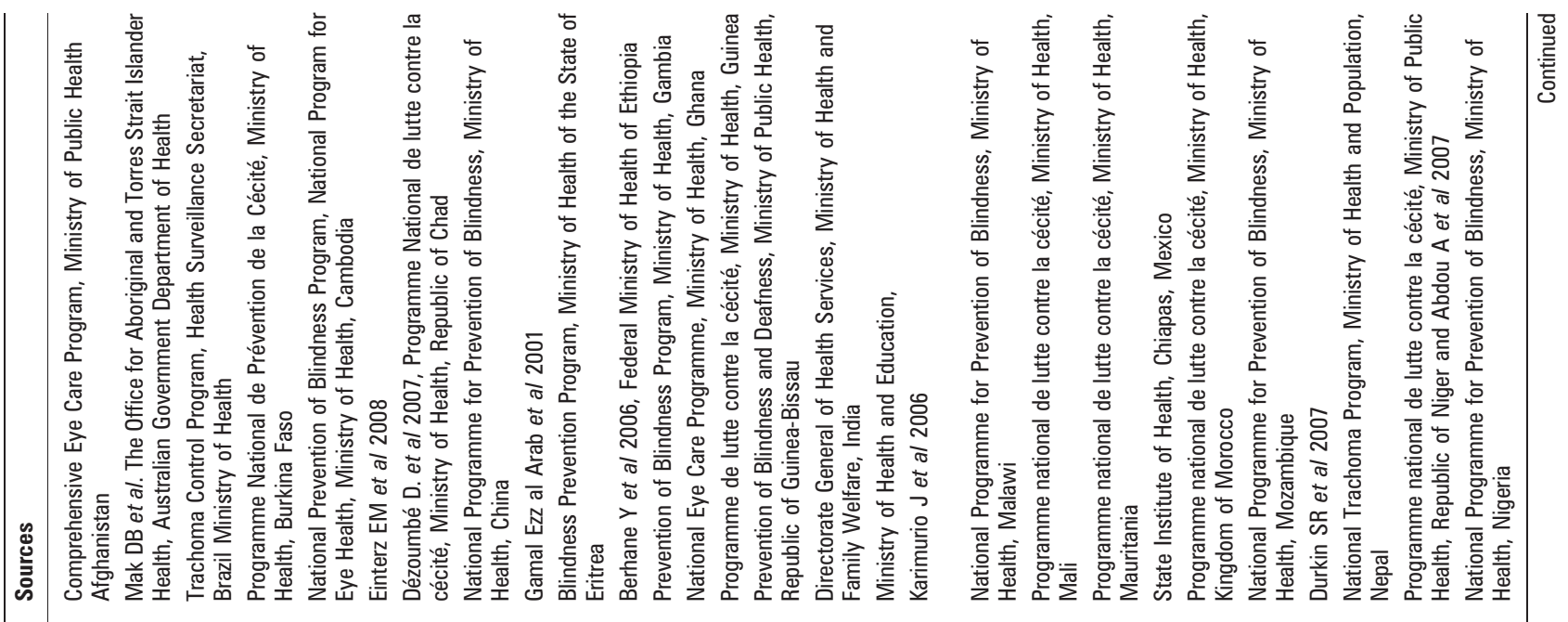

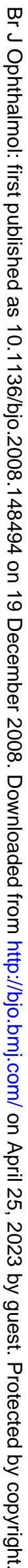

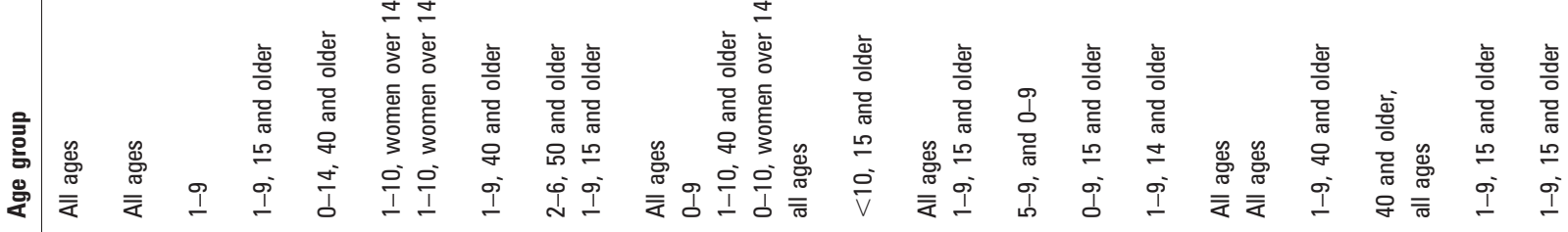
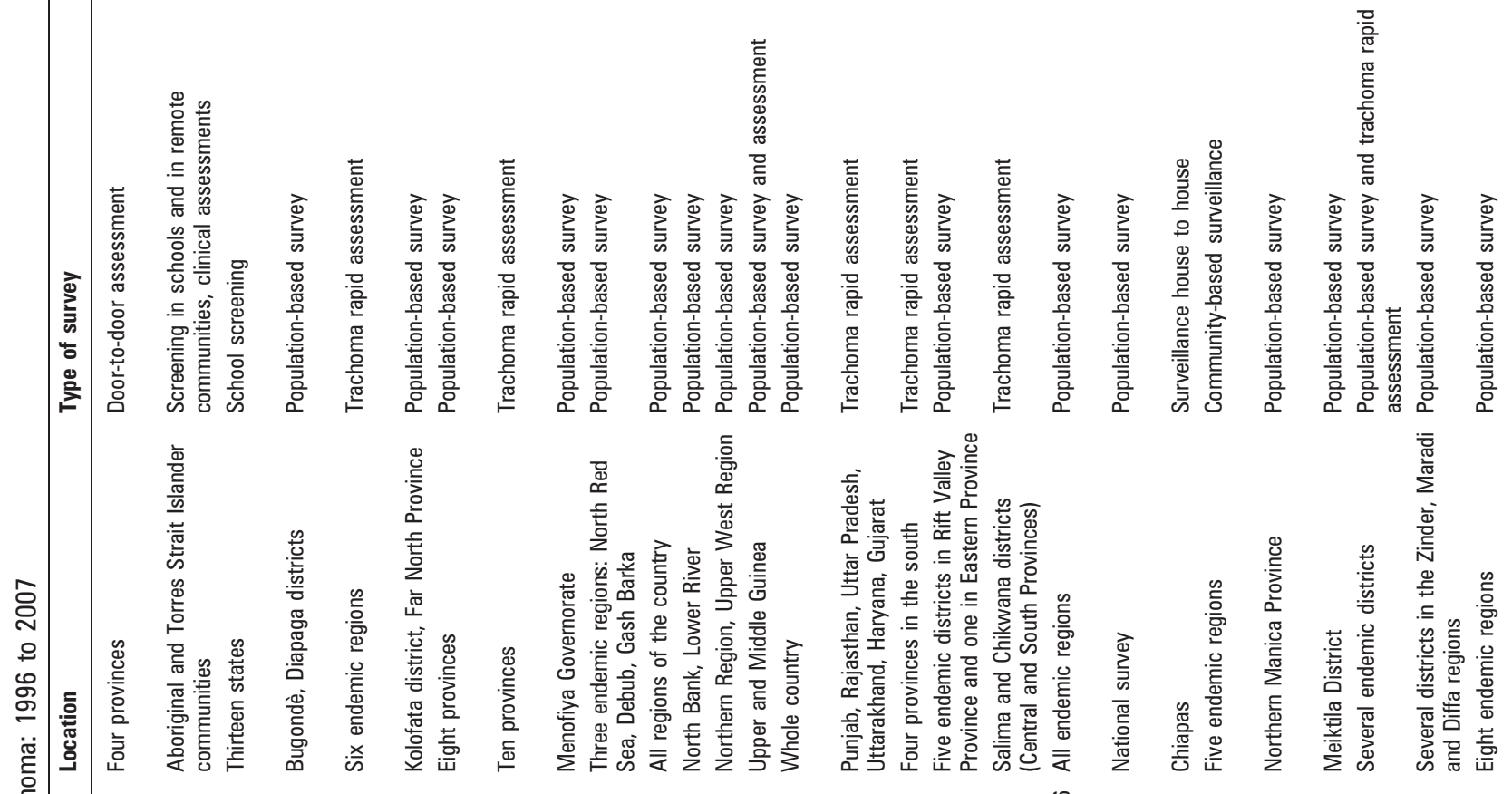

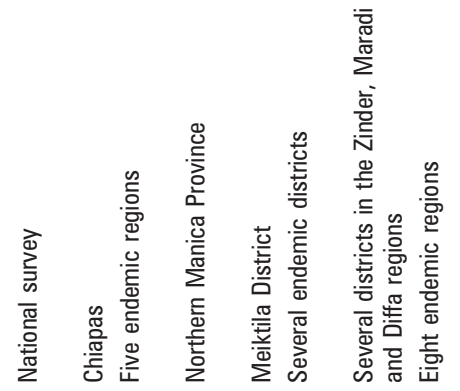

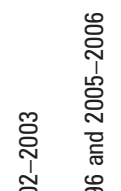

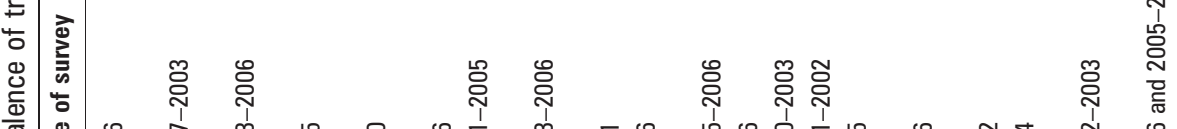

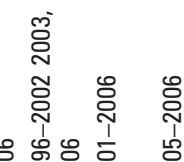

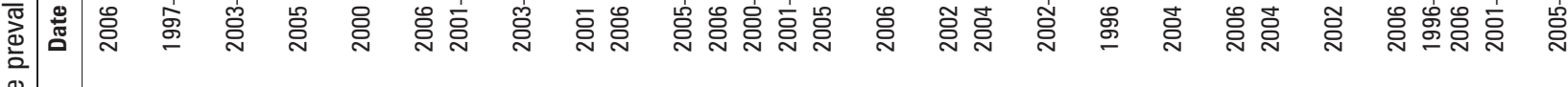

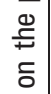

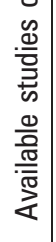

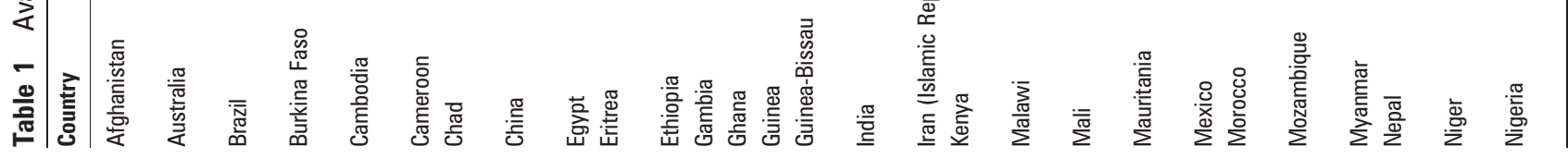




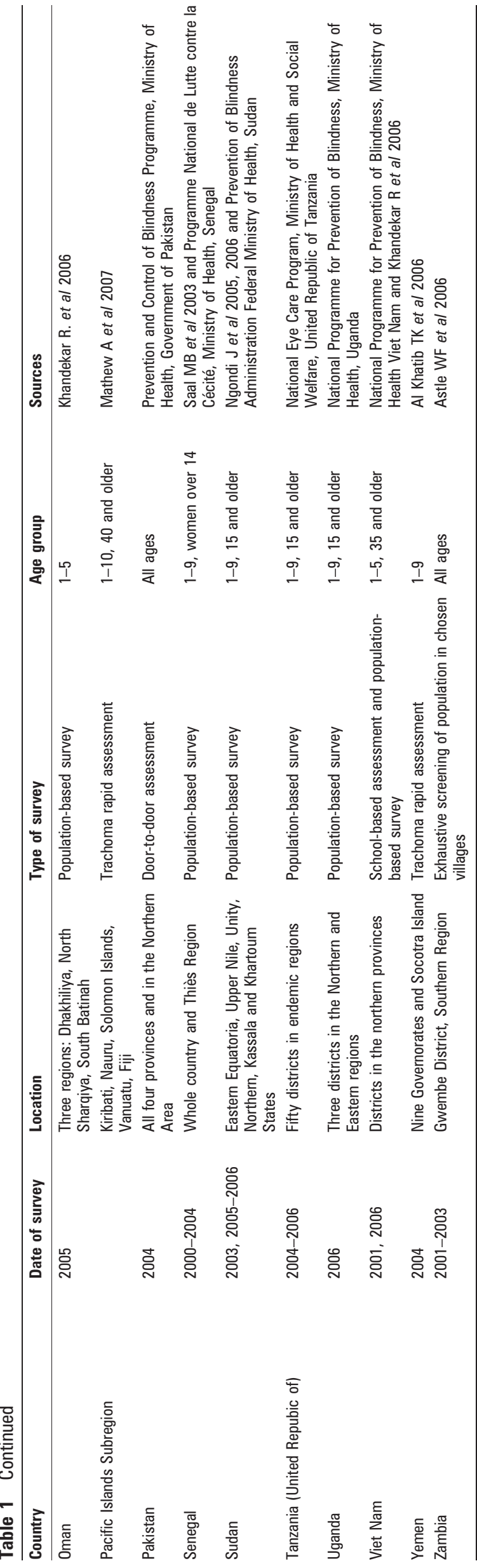

Trichiasis (TT) was defined as at least one eyelash rubbing on the eyeball. Active trachoma was defined as Trachomatous Inflammation-Follicular and/or Trachomatous InflammationIntense (TF/TI).

A database was created to record and analyse country-specific data on prevalence of active trachoma and trichiasis.

\section{Populations}

Estimates of country population were based on the 2004 UN demographic assessment by the United Nations Population Division, ${ }^{12}$ urban and rural distribution from the UN World Urbanization Prospect..$^{13}$ The population size and structure for districts, provinces or states within countries were based on country census when available or from other public domain sources (http://www.geohive.com, http://world-gazetteer.com).

\section{Estimates of prevalence}

Prevalence of active trachoma was estimated from the available data for children less than 10 years old. The prevalence of active trachoma for all ages was estimated using the model proposed at the WHO 2nd Global Scientific Meeting on Trachoma in $2003^{6}$ that was based on the results from the available data from countries and that has been validated in subsequent studies from Gambia, ${ }^{11}$ Ethiopia ${ }^{14}$ and Tanzania. ${ }^{15}$ The model applies four correction factors to the prevalence of TF/TI $<10$ years to make projections for all ages (1.0, 1.1, 1.2, 1.3 to prevalence of $<10 \%, 10-19 \%, 20-29 \%, \leqslant 30 \%$ respectively). The same model provides three correction factors to estimate the prevalence of trichiasis for all the population 14 years and older: 1.3 to be applied to the prevalence determined for women ages 14 and older; 1.05 to age-specific prevalence determined for both sexes over 30 and 1.1 to age-specific prevalence determined for both sexes over 40.

Prevalence was first determined at district level; the results from a single survey within a district were extrapolated to the entire district if it was considered representative and if it was known that the whole district was endemic. Prevalence estimated for one district in a province/state was extrapolated to the whole province/state using analogous criteria.

The total number of cases of trachoma in the country was then estimated from the prevalence in districts, provinces or states; information from country experts was essential to identify the endemic areas and thus avoid over- or underestimates. In a few cases, national surveys were available (see table 1).

In the case of countries missing data, prevalence was estimated from the information in unpublished reports from Ministries of Health or from proxy countries chosen according to similarity of epidemiology and demographic structure.

Based on the prevalence of active trachoma and trichiasis, the Ultimate Intervention Goals (UIG) for Antibiotics and for Surgery were estimated for each country. The UIG-A is the number of people to be treated with antibiotics. In low endemic districts, where the prevalence of active trachoma is less than or equal to $10 \%$, it is the number of cases multiplied by a factor of 3 to include the average number of family members; in high endemic districts, with a prevalence higher than $10 \%$, mass drug treatment is required, and consequently the UIG-A corresponds to the population in the district. The UIG-S ultimate intervention goal for trichiasis surgery is to provide surgical interventions to all current estimated patients. 
Table 2 Estimated active trachoma and trachomatous trichiasis in endemic countries in 2007 from available data

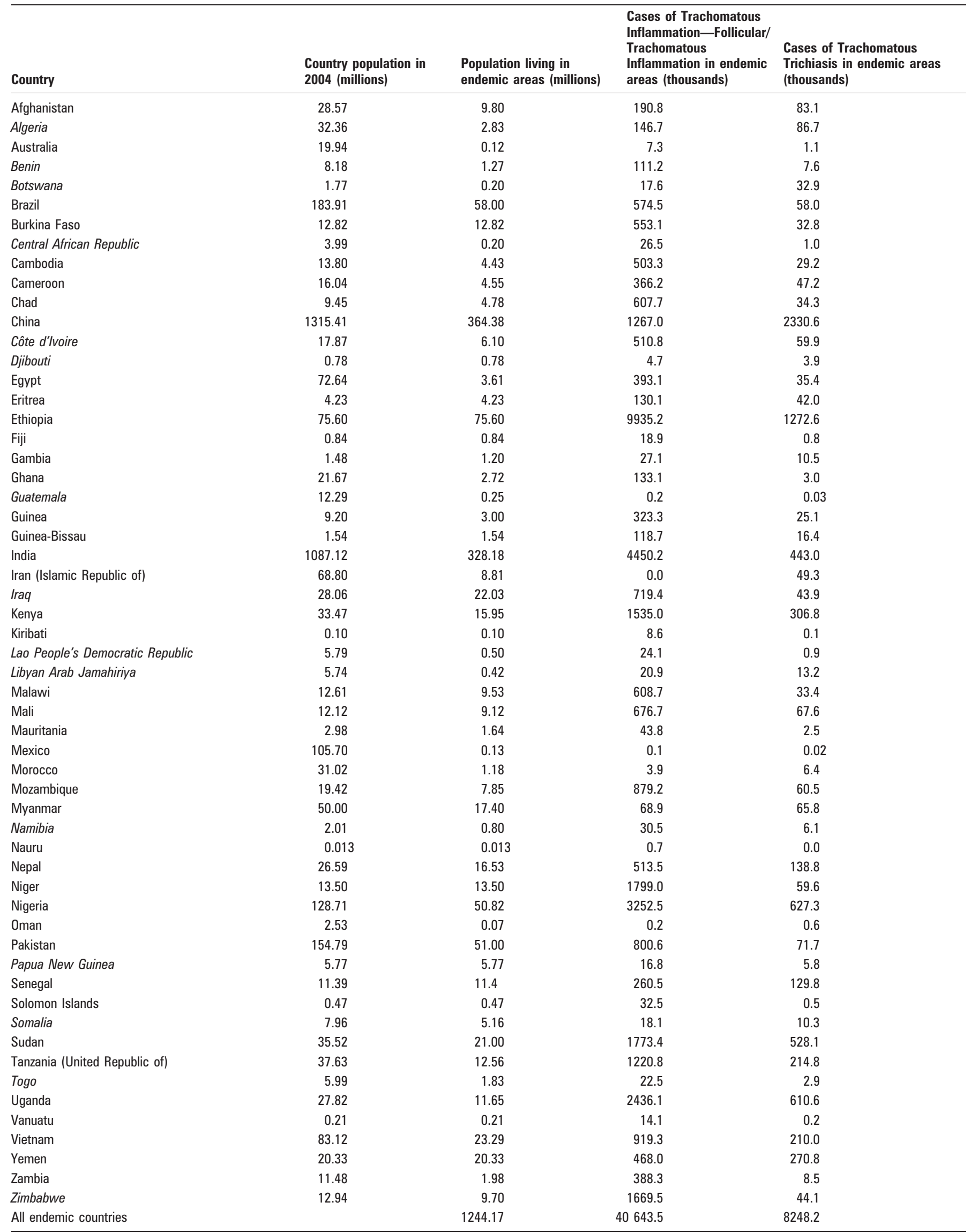

Italics indicate countries for which prevalence was estimated on proxy countries or on other assumptions because of the lack of recent population-based data. 


\section{RESULTS}

For 42 countries out of the 57 known to be endemic for trachoma, there were population-based surveys or assessments consistent with the inclusion criteria for this study. Notably, 32 countries have carried out in the last 5 years national surveys or surveys of entire endemic areas, among them the most populous of the endemic countries, China, India and Nigeria.

For 11 of the known endemic countries (Benin, Botswana, Côte d'Ivoire, Guatemala, Iraq, Libyan Arab Jamahiriya, Namibia, Papua New Guinea, Somalia, Togo and Zimbabwe) there were no surveys conducted in the last 15 or 20 years; for four countries (Algeria, Central African Republic, Djibouti and Lao People's Democratic Republic) the information was prior to the year 2000. In these 15 countries, the estimated population living in endemic areas for trachoma is 58 million, only $22 \%$ of the total population. The available studies for 42 countries are shown in table 1 (for the bibliography, see http://www.who. int/blindness/publications/references/en/trachoma_08.pdf).

Table 2 shows for the 57 endemic countries the estimated population living in endemic areas, the number of people with active trachoma and trichiasis.

Globally, 1.2 billion people live in endemic areas, 40.6 million people are suffering from active trachoma, and 8.2 million have trichiasis; $48.5 \%$ of the global burden of active trachoma is concentrated in five countries: Ethiopia, India, Nigeria, Sudan and Guinea. On the other hand, 50\% of the global burden of trichiasis is concentrated in only three countries: China, Ethiopia and Sudan.

Overall, Africa is the most affected continent; 27.8 million cases of active trachoma ( $68.5 \%$ of all) and 3.8 million cases of trichiasis (46.6\% of all) are located in 28 of the 46 countries in the WHO African Region, with an estimated population of 279 million living in endemic areas. The disease is still prevalent in some countries or areas of countries of four other WHO regions: the Eastern-Mediterranean (12 countries, population in endemic areas 144 million), the Western Pacific (11 countries, population in endemic areas 400 million), South-East Asia (three countries, population in endemic areas 362 million) and the Americas (three countries, population in endemic areas 58 million). The European region is the only WHO region to be free of trachoma. According to these estimates, the ultimate intervention goals require antibiotic treatment for some 340 million people and trichiasis surgeries for 8.2. Trichiasis, however, continues to occur in adults exposed to trachoma in the past: the UIG for trichiasis surgery determined in this paper does not make projections on the incident cases and is therefore an underestimate.

\section{DISCUSSION \\ Limitations}

The estimates presented in this paper are based partly on population-based surveys and assessments, and partly on assumptions and projections. Each of these sources can introduce errors on the final estimates due to the following factors: heterogeneity of sampling and examination methods despite the use of the same WHO protocol; use of a model to estimate prevalence of active trachoma and trichiasis for all ages and for both sexes; assumptions on the prevalence and geographical distribution of trachoma to extrapolate data from communities to districts, from districts to provinces/states and from states/provinces to countries; assumptions to extrapolate prevalence from proxy countries to countries missing data.

To minimise the bias introduced by the limitations above, studies were chosen only if they were consistent with the selection criteria. The projections for all ages and sex were compared with the results from studies that reported age- and sex-specific data, and were found to be consistent. All extrapolations made for countries or areas within a country were based on information from country experts or academic researchers.

Given the heterogeneity of the data and in some cases the non-reporting of the confidence intervals of the studies, it was not possible to determine the extent of uncertainty of the estimates at global level.

\section{Estimated prevalence of active trachoma and trichiasis}

The estimate of 40 million cases of active trachoma presented in this paper is significantly lower than the previous WHO estimate of 84 million made in 2003. ${ }^{6}$ This difference can be attributed to previous overestimations for China and India, but it is also due to declines in prevalence attributable to stable socio-economic development in some countries (eg, Oman, Iran, Vietnam) and in the success of current interventions in other countries where development is still lagging (eg, Mexico, Morocco, The Gambia, Ghana, Mali, etc).

In China and India, reassessment of prevalence was conducted recently. The estimate made in 2004 for China of 26 million cases of active trachoma was based on partial and probably dated figures from the endemic provinces: current estimates are based on population-based assessments conducted in 10 provinces in 2005, Beijing, Sichuan, Yunnan, Oinghai, Chongqing, Shanxi, Zhanjiang, Neimeng, Hainan and Hebei. The standardised prevalence of active trachoma for these provinces was $2.34 \%$ in children aged 10 years and younger. In the absence of data suggesting active trachoma or trichiasis in other provinces, the prevalence was applied only to the population in these provinces. The number of 1.3 million cases of active trachoma therefore could be a conservative estimate.

In India, the previous estimate for 2004 was based on extensive surveys conducted in 1989 in 15 states and one pilot study conducted in 1995. According to the pilot study, there was a $25 \%$ reduction in prevalence of trachoma in India since 1989. Applied to the prevalence values of 1989, this reduction resulted in an estimated 20 million cases of active trachoma. The current estimate is based on the population-based assessments conducted in 2006 in six states, Punjab, Rajasthan, Uttar Pradesh, Uttarakhand, Haryana and Gujarat, that were identified as possibly the only endemic areas in the country. The assessments showed an average prevalence of active trachoma of $6 \%$ in children under 10 years of age resulting in 4.5 million cases when applied to the five endemic states.

Trichiasis is perhaps the most important measure of the severity of the disease because of increased risk of visual impairment. The present estimate of 8.2 million cases of trichiasis exceeds the estimated 7.6 million in 2004: this increase is due to a revision of the estimates according to new available data.

\section{CONCLUSION}

Despite the limitations, this study represents an updated global estimate of trachoma. It is clear that trachoma remains an important public health concern with millions still suffering from an eliminable disease. This study also shows that trachoma remains endemic in many parts of Africa and Asia, and still affects many in Latin American and the Middle East, highlighting the needs and gaps in the provision of services. Obtaining the ultimate intervention goals for antibiotic 
treatment and trichiasis surgeries clearly will require calling for action to governments, NGOs, private sectors and all other health professionals. The SAFE integrated approach recommended by WHO to reach elimination of trachoma by the year 2020 must continue to be implemented and expanded, targeting social development along with healthcare interventions.

Competing interests: SPM and DP are staff members of the World Health Organization. The authors alone are responsible for the views expressed in this publication, and they do not necessarily represent the decisions, policy or views of the World Health Organization.

\section{REFERENCES}

1. Resnikoff S, Pascolini D, Etya'ale D, et al. Global data on visual impairment in the year 2002. Bull World Health Organ 2004;82:844-51.

2. Bowman RJ, Jatta B, Cham B, et al. Natural history of trachomatous scarring in The Gambia: results of a 12-year longitudinal follow-up. Ophthalmology 2001;108:2163-4.

3. Polack S, Brooker S, Kuper H, et al. Mapping the global distribution of trachoma. Bull World Health Organ 2005;83:913-19.

4. West S, Nguyen MP, Mkocha $\mathrm{H}$, et al. Gender equity and trichiasis surgery in the Vietnam and Tanzania national trachoma control programmes. Br J Ophthalmol 2004;88:1368-71.

5. Courtright $\mathbf{P}$, West SK. Contribution of sex-linked biology and gender roles to disparity with trachoma. Emerg Infect Dis 2004;10:2012-16.
6. WHO Alliance for the Global Elimination of Blinding Trachoma by $\mathbf{2 0 2 0 .}$ Report of the 2nd Global Scientific Meeting on Trachoma, Geneva, 25-27 August 2003. Geneva: WHO, 2003 (WHO/PBD/GET.03.1).

7. Frick KD, Basilion EV, Hanson $\mathrm{CL}$, et al. Estimating the burden and economic impact of trachomatous visual loss. Ophthalmic Epidemiol 2003;10:121-32.

8. Prevention of Blindness and Deafness. Planning for the global elimination of trachoma (GET). Report of a WHO consultation. Geneva, 25-26 November 1996. Geneva: WHO, 1997 (WHO/PBL/97.60). http://www.who.int/pbd/publications/ trachoma/en/get 1996.pdf (accessed 6 Feb 2009).

9. Mariotti SP, Prüss A. The SAFE strategy. Preventing trachoma: a guide for environmental sanitation and improved hygiene. Geneva: WHO, 2001 (WHO/PBD/GET/ 00.7/rev.1). http://www.who.int/blindness/SAFE_en.pdf (accessed 6 Feb 2009).

10. Gambir M, Basanez M, Turner F, et al. Trachoma: transmission, infection and control. Lancet Infect Dis 2007;7:420-7.

11. WHO Alliance for the Global Elimination of Blinding Trachoma by $\mathbf{2 0 2 0 .}$ Report of the 11th meeting of the WHO Alliance for the Global Elimination of Blinding Trachoma, 2-4 April 2007, Cairo. Geneva: WHO, 2007 (WHO/PBD/GET/11).

12. United Nations. World population prospects: the 2004 revision (CD-ROM edn, extended dataset). New York: United Nations, 2005

13. United Nations. World urbanization prospects: the 2003 revision. New York: United Nations, 2004.

14. Federal Ministry of Health of Ethiopia. National survey on blindness, low vision and trachoma in Ethiopia. Addis Ababa: Federal Ministry of Health of Ethiopia, 2006.

15. Solomon AW, Holland MJ, Alexander NDE, et al. Mass treatment with single-dose azithromycin for trachoma. N Engl J Med 2004;351:1962-71. 